\title{
Integral quantum Hall effect in graphene: Zero and finite Hall field
}

\author{
P. M. Krstajić and P. Vasilopoulos \\ Concordia University, Department of Physics, 7141 Sherbrooke Ouest, Montréal, Québec, Canada H4B 1R6
}

(Received 1 September 2010; revised manuscript received 2 December 2010; published 23 February 2011)

\begin{abstract}
We study the influence of a finite Hall field $E_{H}$ on the Hall conductivity $\sigma_{y x}$ in graphene. Analytical expressions are derived for $\sigma_{y x}$ using the Kubo-Greenwood formula. For vanishing $E_{H}$, we obtain the well-known expression $\sigma_{y x}=4(N+1 / 2) e^{2} / h$. The inclusion of the dispersion of the energy levels, previously not considered, and their width, due to scattering by impurities, produces the plateau of the $n=0$ Landau level. Further, we evaluate the longitudinal resistivity $\rho_{x x}$ and show that it exhibits an oscillatory behavior with the electron concentration. The peak values of $\rho_{x x}$ depend strongly on the impurity concentration and their potential. For a finite $E_{H}$, the result for $\sigma_{y x}$ is the same as that for $E_{H}=0$, provided $E_{H}$ is not strong, but the values and positions of the resistivity maxima are modified due to the $E_{H}$-dependent dispersion of the energy levels.
\end{abstract}

DOI: 10.1103/PhysRevB.83.075427

PACS number(s): 71.10.Pm, 72.25.-b, 73.21.-b

\section{INTRODUCTION}

The crystal structure of graphene, a single atomic layer of graphite, can be viewed as two interpenetrating triangular sublattices. ${ }^{1}$ Charge carriers in a wide single-layer graphene behave like "relativistic," chiral massless particles with a "light speed" equal to the Fermi velocity and possess a gapless spectrum that is linear in the wave vector near the $K$ and $K^{\prime}$ points. One major consequence is the perfect transmission through arbitrarily high and wide barriers, referred to as Klein tunneling (see a recent review in Ref. 1).

When a doped graphene sheet is sufficiently clean and is subject to high magnetic fields, it exhibits a half-integer quantum Hall effect (IQHE), $\sigma_{y x}=4(N+1 / 2) e^{2} / h$, that is very different from the conventional one in $\mathrm{Si}$ or GaAs/AlGaAs heterostructures, $\sigma_{y x}=2(N+1) e^{2} / h$, with $N$ being the largest integer contained in $E_{F} / \hbar \omega_{c}, E_{F}$ the Fermi energy, and $\hbar \omega_{c}$ the cyclotron energy. This can be attributed to massless Dirac fermions in this material, as theoretically predicted by several authors. ${ }^{2-4}$ It should be mentioned, however, that the existence of this quantum state in planar and gapless semiconductors was previously suggested by Schakel $^{5}$ by studying quantum electrodynamics in $(2+1)$ dimensions at finite density and temperature. It was first observed in 2005 at low temperatures, ${ }^{6,7}$ and later even at room temperature, due to the high cyclotron energy $\hbar \omega_{c}$ compared to thermal energy $k_{B} T .{ }^{8}$ Since then several experiments have confirmed not only the existence of this quantum state in graphene, ${ }^{9-11}$ but also the appearance of additional plateaus at very high fields ${ }^{12,13}$ due to the lifting of the degeneracy of the spectrum. On the other hand, the precision of the quantization of the first two plateaus was determined ${ }^{7}$ to be 1 in $10^{4}$ for the Hall resistivity $\rho_{x y}$.

To date, there have been several theoretical treatments ${ }^{14-19}$ devoted to the IQHE in graphene. Apparently, the IQHE can be attributed to several causes; see especially Refs. 17 and 18, and references cited therein. One of the possible causes, consistent with many experimental observations, is scattering by impurities; another one is electron-electron interaction. According to Ref. 17, scattering by long-range disorder, e.g., Coulomb impurities, is supported by experimental observations (see also Refs. 2, 3, and 20). However, to date there is no definitive answer as to what the cause of the IQHE is, and other treatments emphasizing smooth, random scalar potentials, percolation of the guiding center, valley mixing, dislocations, etc., have appeared. ${ }^{17,18,20}$

A simple and general approach to the IQHE in graphene is to evaluate the Kubo formula (see, e.g., Refs. 2, 3, and 20) in the self-consistent Born approximation. Although this approximation fails for smooth, random potentials, ${ }^{21}$ the simplicity of the formula is attractive and gives results in line with experimental observations. In this paper, using Kubo formulas expressed explicitly in terms of one-particle eigenstates, we investigate the influence of both a finite and a negligible Hall field $E_{H}$ on the Hall conductivity in graphene, and show that the plateau of the $n=0$ Landau level can be accounted for by the dispersion of the energy levels due to scattering by impurities. In addition, we evaluate the longitudinal resistivity $\rho_{x x}$ for scattering by short- and long-range impurities.

The influence of a finite Hall field $E_{H}$ follows a treatment previously applied to the breakdown of the usual IQHE ${ }^{22}$ by including $E_{H}$ in the one-electron Hamiltonian. It was justified in Ref. 23 and accounts for electron-electron interactions in the Hartree approximation. ${ }^{24}$ The result is the analytical expressions for the eigenvectors and eigenvalues in crossed electric and magnetic fields in graphene given in Refs. 25 and 26. We use these results to evaluate the relevant conductivities.

In Sec. II we present the model and evaluate the Hall conductivity $\sigma_{y x}$ and the longitudinal conductivity $\sigma_{x x}$ for a finite Hall field $E_{H}$. We then present results for a negligible field $E_{H}$ in Sec. III, discuss the lifting of degeneracies due to scattering in Sec. IV, and make concluding remarks in Sec. V.

\section{FINITE HALL FIELD}

The one-electron Hamiltonian for a graphene layer in the presence of a magnetic field $B$ perpendicular to the layer, while the Hall field $E_{H}$ is in the plane, reads

$$
H=v_{F} \boldsymbol{\sigma}(\mathbf{p}+e \mathbf{A})-e E_{H} y \mathbf{1},
$$

where $\mathbf{1}$ is the unit $2 \times 2$ matrix. The Hamiltonian given by Eq. (1) is written in $(2+1)$ dimensions. We adopt the Landau gauge $\mathbf{A}=(-B y, 0)$, with the magnetic field oriented along the positive $z$ axis. The Zeeman splitting is justifiably neglected for moderate values of the field $B$. The dimensionless 
parameter $\eta=E_{H} / v_{F} B$ characterizes the strength of the Hall field. The problem of the crossed electric and magnetic fields has been solved analytically by Lukose et al. ${ }^{25}$ and by Peres et $a l{ }^{26}$ Here the electric field is the Hall field $E_{H}$ and the eigenvectors are

$$
|\Psi\rangle_{ \pm}=\sqrt{\frac{\eta}{4}}\left(\begin{array}{c}
-\sqrt{C_{+}} \Phi_{n-1} \mp \sqrt{C_{-}} \Phi_{n} \\
\sqrt{C_{-}} \Phi_{n-1} \pm \sqrt{C_{+}} \Phi_{n}
\end{array}\right) e^{i k_{x} x},
$$

where $C_{ \pm}=(1 \pm \sqrt{\gamma}) / \eta ; \Phi_{n}=\Phi_{n}\left(\xi^{\prime}\right)$ are the usual oscillator functions, with

$$
\xi^{\prime}=\left[y-l_{c}^{2} k_{x}-s \sqrt{2 n} l_{c} \eta / \gamma^{1 / 4}\right] \gamma^{1 / 4} / l_{c} ;
$$

$l_{c}$ is the magnetic length $l_{c}=\sqrt{\hbar / e B}$; and $\gamma=1-\eta^{2}$. The center of the orbit is $y_{c}=l_{c}^{2} k_{x}+s \sqrt{2 n} l_{c} \eta / \gamma^{1 / 4}$. The eigenvalues of the Hamiltonian (1) are

$$
E_{s n k_{x}}=s \sqrt{n} \hbar \omega_{c} \gamma^{3 / 4}-e E_{H} l_{c}^{2} k_{x},
$$

where $s$ takes values $s=+1,-1$ for the $\pi$ and $\pi^{*}$ band, respectively, and $\omega_{c}=\sqrt{2} v_{F} / l_{c}$. However, the $n=0$ level must be treated separately. Its eigenvalue is the second term in Eq. (4) and its eigenvector, corresponding to the Dirac point, is

$$
|\Psi\rangle_{0}=\sqrt{\frac{\eta}{2}}\left(\begin{array}{c}
-\sqrt{C_{-}} \\
\sqrt{C_{+}}
\end{array}\right) \Phi_{0} e^{i k_{x} x} .
$$

We now consider linear responses to a weak source-to-drain electric field. With linear response theory, the corresponding Hall conductivity $(\mu=x, y ; \nu=x, y ; \mu \neq v)$ is given by ${ }^{27}$

$$
\begin{aligned}
\sigma_{\mu \nu}^{n d}= & \frac{i \hbar e^{2}}{S_{0}} \sum_{\zeta \neq \zeta^{\prime}} f_{\zeta}\left(1-f_{\zeta^{\prime}}\right) v_{\nu \zeta \zeta^{\prime}} v_{\mu \zeta^{\prime} \zeta} \\
& \times \frac{1-e^{\beta\left(E_{\zeta}-E_{\zeta^{\prime}}\right)}}{E_{\zeta}-E_{\zeta^{\prime}}} \lim _{\epsilon \rightarrow 0} \frac{1}{E_{\zeta}-E_{\zeta^{\prime}}+i \epsilon} .
\end{aligned}
$$

Here $S_{0}$ is the area, $v_{v, \mu \zeta \zeta^{\prime}}$ are the matrix elements of the velocity operator $v_{v}$, and $f_{\zeta}=f\left(E_{\zeta}\right)$ is the Fermi-Dirac distribution function. If we use the identity $f_{\zeta}\left(1-f_{\zeta^{\prime}}\right) \exp \left[\beta\left(E_{\zeta}-\right.\right.$ $\left.\left.E_{\zeta^{\prime}}\right)\right]=f_{\zeta^{\prime}}\left(1-f_{\zeta}\right)$, and replace $\epsilon$ by the level width $\Gamma_{\zeta}$, Eq. (6) becomes the well-known Kubo-Greenwood formula,

$$
\sigma_{\mu \nu}^{n d}=\frac{i \hbar e^{2}}{S_{0}} \sum_{\zeta, \zeta^{\prime}} \frac{\left(f_{\zeta}-f_{\zeta^{\prime}}\right) v_{\nu \zeta \zeta^{\prime}} v_{\mu \zeta^{\prime} \zeta}}{\left(E_{\zeta}-E_{\zeta^{\prime}}\right)\left(E_{\zeta}-E_{\zeta^{\prime}}+i \Gamma_{\zeta}\right)} .
$$

The sum is over all quantum numbers $|\zeta\rangle=\left|s, n, k_{x}\right\rangle$ and $\left|\zeta^{\prime}\right\rangle=\left|s^{\prime}, n^{\prime}, k_{x}^{\prime}\right\rangle$, with $|\zeta\rangle \neq\left|\zeta^{\prime}\right\rangle$. If the broadening $\Gamma_{\zeta}$ is the same for all states $|\zeta\rangle$, it can be proven that the imaginary part of the whole expression in Eq. (7) vanishes identically. Then the denominator in Eq. (7) can be replaced by $\left(E_{\zeta}-\right.$ $\left.E_{\zeta^{\prime}}\right)^{2}+\Gamma_{\zeta}^{2}$ (see Appendix A). In what follows, though, we first take $\Gamma_{\zeta}=0$. Later we will assume $\Gamma_{\zeta} \approx \Gamma$, and estimate the resulting corrections. The broadening $\Gamma$ is much larger than in conventional semiconductors and depends relatively weakly on the temperature.

In the spirit of Refs. 23 and 22, we assume that a finite field $E_{H}$ affects only the scattering of electrons by collisions with impurities and not the Fermi functions that enter Eq. (7); that is, we assume equilibrium electron distributions, at least for a relatively weak $E_{H}$. After all, we consider linear responses to a weak, source-to-drain electric field and the approach is previously justified..$^{22,23,28}$

In graphene there are two unequivalent points $\left(K, K^{\prime}\right)$ in the Brillouin zone due to the two sublattices and, with negligible Zeeman splitting, the degeneracy is fourfold, $g_{s}=4$. This degeneracy is lifted only at very high magnetic fields ${ }^{13}$ $B>20 \mathrm{~T}$; this case will not be considered in this work. The matrix elements of the velocity operator between the states $\left|s n k_{x}\right\rangle$ and $\left|s^{\prime} n^{\prime} k_{x}^{\prime}\right\rangle$ are diagonal in $k_{x}\left(\propto \delta_{k_{x} k_{x}^{\prime}}\right)$. With $k_{x}$ and $k_{x}^{\prime}$ suppressed, they read

$$
\left\langle s n\left|v_{x}\right| s^{\prime} n^{\prime}\right\rangle=-\frac{v_{F}}{2}\left[\left(1+s s^{\prime}\right) \delta_{n, n^{\prime}}+s^{\prime} \delta_{n-1, n^{\prime}}+s \delta_{n, n^{\prime}-1}\right],
$$

$$
\left\langle s^{\prime} n^{\prime}\left|v_{y}\right| s n\right\rangle=-i\left(v_{F} / 2\right) \sqrt{\gamma}\left(-s^{\prime} \delta_{n-1, n^{\prime}}+s \delta_{n, n^{\prime}-1}\right) .
$$

Their product is

$$
P_{n n^{\prime}}^{s s^{\prime}}=i\left(v_{F}^{2} / 4\right) \sqrt{\gamma}\left(-\delta_{n-1, n^{\prime}}+\delta_{n, n^{\prime}-1}\right) .
$$

However, the matrix elements between the zeroth state $(n=0$ or $n^{\prime}=0$ ) and any other state should be evaluated separately. For example,

$$
\left\langle 0\left|v_{x}\right| s^{\prime} n^{\prime}\right\rangle=-\left(v_{F} / \sqrt{2}\right) \delta_{0, n^{\prime}-1} .
$$

Note that the boundary condition restricts $k_{x}$ in the range $-L_{y} / 2 l_{c}^{2}-k_{0} \leqslant k_{x} \leqslant L_{y} / 2 l_{c}^{2}-k_{0}$, where $k_{0}=$ $s \sqrt{2 n} \eta / \gamma^{1 / 4} l_{c}$. After summation over $k_{x}$ and $k_{x}^{\prime}$, the general term of the sum in Eq. (7), denoted as $A_{n n^{\prime}}^{s s^{\prime}}$, reads

$$
A_{n n^{\prime}}^{s s^{\prime}}=\frac{e^{2} g_{s}}{8 h \gamma} \frac{f_{n}^{s}-f_{n^{\prime}}^{s^{\prime}}}{\left(s \sqrt{n}-s^{\prime} \sqrt{n^{\prime}}\right)^{2}}\left(\delta_{n-1, n^{\prime}}-\delta_{n, n^{\prime}-1}\right),
$$

where $f_{n}^{s}=f_{n}^{s}\left(E_{s n 0}\right)$ is the Fermi-Dirac distribution function. On the other hand, to determine the position of the Fermi level $E_{F}$, one needs the density of states, which for the case of graphene subject to perpendicular magnetic and electric fields reads

$$
D(E)=\frac{g_{s}}{h} \frac{e B}{V_{H}} \sum_{n, s}\left[\theta\left(E_{+}-E_{s n 0}^{\prime}\right) \theta\left(E_{-}+E_{s n 0}^{\prime}\right)\right],
$$

with $\theta$ the Heaviside function, $E_{ \pm}=V_{H} / 2 \pm E$, and $E_{s n 0}^{\prime}=$ $E_{s n 0}+e E_{H} l_{c}^{2} k_{0}$. When the Hall field approaches zero $\left(E_{H} \rightarrow\right.$ $0)$, the density of states turns into a series of $\delta$ functions centered at $E_{s n 0}^{0}=s \sqrt{n} \hbar \omega_{c}$, with the factor $g_{s} e B / h$ as the $k_{x}$ degeneracy. However, in this paper, we will adopt the commonly used ${ }^{14}$ Gaussian-like density of states, with a constant width $\Gamma$, to account for the broadening due to impurities.

We proceed with the evaluation of $\sigma_{y x}^{n d}$, i.e., with the summation of the terms $A_{n n^{\prime}}^{s s^{\prime}}$ in Eq. (7). In order to arrive at neat expressions, one may notice that grouping terms such as $A_{n n^{\prime}}^{++}$ and $A_{n n^{\prime}}^{+-}$together that contain $f_{n}^{+}$leads to the cancellation of $2 \sqrt{n(n+1)}$ factors. The same holds for the $A_{n n^{\prime}}^{--}$and $A_{n n^{\prime}}^{-+}$ terms. At this point, special care should be taken for the $n=0$ level, since its degeneracy with respect to a particular electron or hole component is twice as small as that of other Landau levels. On the other hand, the matrix elements of the velocity operators that involve the $n=0$ level are larger by a factor of $\sqrt{2}$ for both $v_{x}$ and $v_{y}$, which compensates for the smaller 
degeneracy of the zeroth level. It means the term $n=0$ can easily be included in the final sum that runs over all values of $n$ and $s$. This yields the Hall conductivity

$$
\sigma_{y x}^{n d}=\frac{4 e^{2}}{h \gamma} \sum_{n}\left(n+\frac{1}{2}\right)\left(f_{n}^{+}-f_{n+1}^{+}+f_{n}^{-}-f_{n+1}^{-}\right) .
$$

Taking into account the identity $\tanh \left[\beta\left(E-E_{F}\right) / 2\right]=1-$ $2 f(E)$, one easily arrives at the same expression for the Hall conductivity for vanishing Hall field as derived in Ref. 14. Further, at small temperatures and large magnetic fields, one may take, for an $n$-type sample, all $f_{i}^{+}(i=0,1, \ldots, N)$ equal to unity, while $f_{N+1}^{+} \approx 0$ for a certain $N$ (where all hole states are occupied, $f_{i}^{-}=1$ ). Then Eq. (14) yields the well-known result

$$
\sigma_{y x}^{n d}=\frac{4 e^{2}}{h}\left(N+\frac{1}{2}\right) .
$$

Notice, however, that the plateaus are not simply determined by the Fermi functions and their thermal broadening - the role of disorder is shown to be very important (see Refs. 17 and 20). An approximate treatment is to assess its influence on the Hall conductivity through the level broadening $\Gamma$ due to scattering by impurities given below in Eq. (17). One can expand the denominator in Eq. (7) in powers of $\Gamma / \hbar \omega_{c}$ (see Appendix B). The first $\Gamma$-dependent term in the expansion is evaluated following the procedure outlined above and gives the correction $\Delta \sigma_{y x}^{n d}$ [cf. Eq. (B5)]. Then the relative deviation of the Hall conductivity is

$$
\frac{\Delta \sigma_{y x}^{n d}}{\sigma_{y x}^{n d}} \cong-\frac{4}{\gamma^{3 / 2}}\left(\frac{\Gamma}{\hbar \omega_{c}}\right)^{2} \frac{N^{2}+N+1 / 8}{N+1 / 2} .
$$

On the other hand, the level width $\Gamma$, obtained in the selfconsistent Born approximation, ${ }^{2}$ is given by

$$
\Gamma=\sqrt{2 / A} \hbar \omega_{c},
$$

where the dimensionless parameter $A$ depends on the density of impurities and their potential. When the scattering is weak, i.e., when the sample is clean, its value ${ }^{2}$ is $A \approx 100$. Then the deviation from the $\Gamma=0$ case, for $B=14 \mathrm{~T}$ and $\gamma \approx 1$, is about $2 \%$ for the $N=0$ level.

In Fig. 1 we show the Hall resistivity $\rho_{x y}$ versus magnetic field for constant electron concentration $n_{e}=10^{12} \mathrm{~cm}^{-2}$. Only results for $E_{H}=0$ are shown since, for appropriate (nonzero) values of $E_{H} \lesssim 20 \mathrm{kV} \mathrm{cm}^{-1}$ and magnetic field $B>2 \mathrm{~T}$, the $E_{H} \neq 0$ results differ from those shown by less than $0.1 \%$.

Next we proceed with the evaluation of the longitudinal conductivity $\sigma_{x x}$ in graphene under crossed magnetic and electric fields. It has been shown ${ }^{29}$ that, only if the scatterers are assumed to be screened, charged impurities, one may expect the conductivity in graphene (without magnetic field) to increase linearly with the electron concentration, in agreement with experimental data. ${ }^{30}$ However, at very small concentrations, the conductivity $\sigma_{x x}$ does not vanish, but bottoms at a value close to $4 e^{2} / h$. Still the value and the physical origin of the conductivity minimum $\sigma_{\min }$ is somewhat a matter of open debate in the literature. Early theoretical work $^{31,32}$ predicted $\sigma_{\text {min }}=4 e^{2} / \pi h$ for vanishing disorder. In the presence of charged impurities, however, the value $\sigma_{\min }=$ $4 e^{2} / h$ is predicted. ${ }^{33,34}$ More recent experimental work $^{35}$

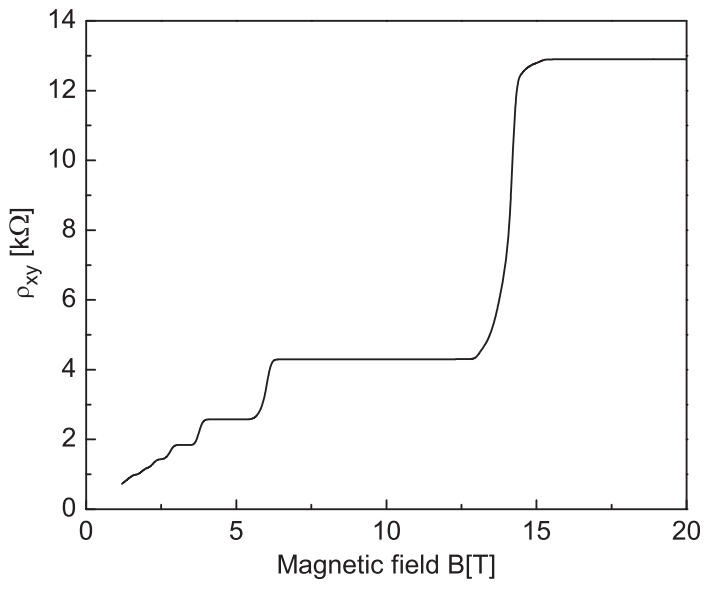

FIG. 1. Hall resistivity $\rho_{x y}$ vs magnetic field $B$ for electron density $n_{e}=10^{12} \mathrm{~cm}^{-2}$.

suggests that the conductivity minimum is not determined by the physics of the Dirac-point singularity, but rather by carrierdensity inhomogeneities induced by charged impurities. All this discussion applies to the case when the magnetic field is absent. In this work, we will adopt the view presented in Ref. 35 and evaluate the longitudinal conductivity $\sigma_{x x}$ assuming it is governed by carrier collisions with charged impurities. In a normal magnetic field, the diffusive contribution $\sigma_{x x}^{\text {dif }}$ to this conductivity usually vanishes ${ }^{36}$ and only the collisional contribution $\sigma_{x x}^{\text {col }}$ is important. This is given by ${ }^{27}$

$$
\sigma_{x x}^{\mathrm{col}}=\frac{\beta e^{2}}{S_{0}} \sum_{\zeta, \zeta^{\prime}} f(\varepsilon)\left[1-f\left(\varepsilon^{\prime}\right)\right] W_{\zeta \zeta^{\prime}}\left(\varepsilon, \varepsilon^{\prime}\right)\left(y_{\zeta}-y_{\zeta^{\prime}}\right)^{2},
$$

where $y_{\zeta}=\langle\zeta|y| \zeta\rangle$, and $W_{\zeta \zeta^{\prime}}\left(\varepsilon, \varepsilon^{\prime}\right)$ is the transition rate in the presence of impurities. If scattering by impurities is assumed to be elastic, with $N_{I}$ being their density, the transition rate $W_{\zeta \zeta^{\prime}}$ acquires the form

$$
\begin{aligned}
W_{\zeta \zeta^{\prime}}\left(\varepsilon, \varepsilon^{\prime}\right)= & \frac{2 \pi N_{I}}{\hbar S_{0}} \sum_{\mathbf{q}}|U(\mathbf{q})|^{2}\left|F_{\zeta \zeta^{\prime}}(u)\right|^{2} \\
& \times \delta\left(\varepsilon-\varepsilon^{\prime}\right) \delta_{k_{x}, k_{x}^{\prime}+q_{x}},
\end{aligned}
$$

where $u=l_{c}^{2}\left(q_{x}^{2}+q_{y}^{2}\right) / 2 \gamma^{1 / 2} . U(\mathbf{q})$ is the Fourier transform of the screened impurity potential $U(\mathbf{r})=\left(e^{2} / 4 \pi \epsilon_{0} \epsilon\right) e^{-k_{s} r} / r$, where $\epsilon$ is the static dielectric constant, $\epsilon_{0}$ is the dielectric permittivity, and $k_{s}$ is the screening wave vector. $F_{\zeta \zeta^{\prime}}(u)$ are the form factors, $\left\langle\zeta\left|e^{i \mathbf{q} \cdot \mathbf{r}}\right| \zeta^{\prime}\right\rangle$. The values of the screening length range from ${ }^{37} 10 \mathrm{~nm}$ to $100 \mathrm{~nm}$, depending on the quality of the sample; the corresponding (extremal) value of $k_{s}=10^{7} \mathrm{~m}^{-1}$ will be mostly used in this work. With $U_{0}=e^{2} / 4 \pi \epsilon_{0} \epsilon$, the transform of $U(\mathbf{r})$ is $U(\mathbf{q})=U_{0} /\left(q^{2}+k_{s}^{2}\right)^{1 / 2}$, where $q^{2}=$ $q_{x}^{2}+q_{y}^{2}$. It can be proven that the expectation value of the $y$ coordinate, i.e., $\langle\zeta|y| \zeta\rangle$, is

$$
\langle\zeta|y| \zeta\rangle=y_{c}+s \eta \sqrt{n / 2} l_{c} / \gamma^{1 / 4}
$$

Due to the presence of the $\delta$ function and the Kronecker $\delta$ in Eq. (19), transitions are possible only between different levels $n \neq n^{\prime}$. The form factors $\left|F_{\zeta \zeta^{\prime}}(u)\right|^{2}$ can be evaluated 
analytically if one neglects the dependence of the center of the orbit on $n$ and $s$,

$$
\begin{aligned}
\left|F_{n n-1}^{s s}(u)\right|^{2} \approx & \frac{\eta^{2} e^{-u}}{4}\left\{\frac{\sqrt{u}}{\eta}\left[\frac{L_{n-2}^{1}(u)}{\sqrt{n-1}}+\frac{L_{n-1}^{1}(u)}{\sqrt{n}}\right]\right. \\
-s & {\left.\left[L_{n-1}(u)+\frac{u L_{n-2}^{2}(u)}{\sqrt{n(n-1)}}\right]\right\}^{2}, }
\end{aligned}
$$

with $L_{n}$ and $L_{n}^{m}$ the Laguerre and associated Laguerre polynomials, respectively. The expression for $\left|F_{n n+1}^{s s}\right|^{2}$ is

$$
\left|F_{n n+1}^{s s}(u)\right|^{2}=\left.\left|F_{n n-1}^{s s}(u)\right|^{2}\right|_{n \rightarrow n+1} \text {. }
$$

As usual, the form factors $\left|F_{01}^{s s}(u)\right|^{2}$ should be treated separately; the result is

$$
\left|F_{01}^{s s}(u)\right|^{2} \approx \eta^{2} e^{-u}\left[L_{0}(u)-(s / \eta) \sqrt{u} L_{0}^{1}(u)\right]^{2} / 2 .
$$

Finally, the form factors between the \pm bands are

$$
\left|F_{n n}^{+-}(u)\right|^{2} \approx e^{-u}\left[L_{n}^{-1}(u)\right]^{2} / 4
$$

With these form factors, Eq. (18) takes the form

$$
\begin{aligned}
\sigma_{x x}^{\mathrm{col}}= & \frac{e^{2}}{h} \frac{\beta U_{0}^{2} N_{I} \gamma^{1 / 2}}{2 \pi \hbar \omega_{c}} \sum_{s, s^{\prime}, n, n^{\prime}} f\left(E_{s n 0}\right)\left[1-f\left(E_{s n 0}\right)\right] \\
& \times \int_{0}^{\infty} d u \frac{u\left|F_{n n^{\prime}}^{s s^{\prime}}(u)\right|^{2}}{u+u_{c s}},
\end{aligned}
$$

where $u_{c s}=l_{c}^{2} k_{s}^{2} / 2 \gamma^{1 / 2}$ and $n^{\prime}=n \pm 1$. Since the gaps between the Landau levels in graphene are of the order of $10^{3} \mathrm{~K}$, the product $\beta f\left(E_{s n}\right)\left[1-f\left(E_{s n}\right)\right]$ in Eq. (25) can be replaced, at low temperatures, by the Dirac $\delta$ function, $\delta\left(E-E_{F}\right)$. The corresponding longitudinal resistivity is shown in Fig. 2 (black solid curve) and will be discussed after Eq. (30).

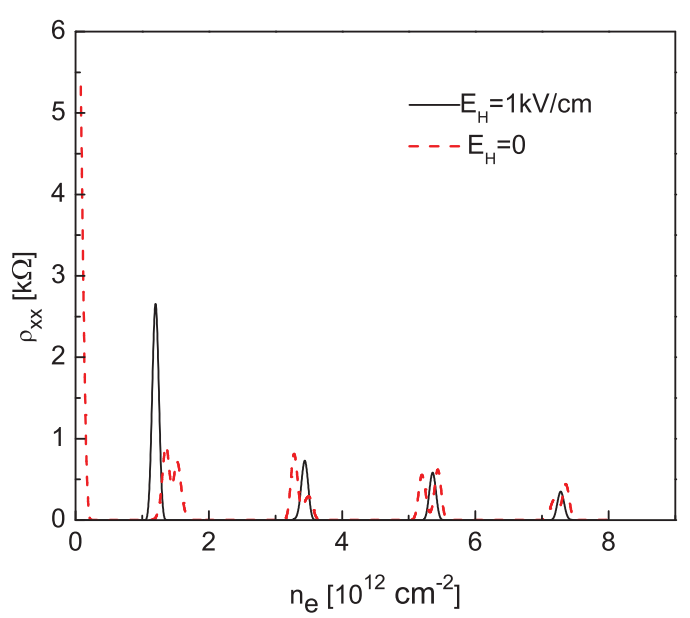

FIG. 2. (Color online) Longitudinal resistivity $\rho_{x x}$ of graphene as a function of the electron concentration $n_{e}$ for Hall field $E_{H}=$ $1 \mathrm{kV} \mathrm{cm}^{-1}$ (black solid curve) and for $E_{H}=0$ (red dashed curve). The other parameters are $A=40$, the screening wave vector $k_{s}=$ $10^{7} \mathrm{~m}^{-1}$, and the impurity concentration $n_{i}=5 \times 10^{10} \mathrm{~cm}^{-2}$.

\section{ZERO HALL FIELD}

When $E_{H}$ is absent, the eigenvectors take the form

$$
|\Psi\rangle=\frac{1}{\sqrt{2}}\left(\begin{array}{c}
\Phi_{n-1} \\
\pm \Phi_{n}
\end{array}\right) e^{i k_{x} x}
$$

and the eigenvalues become $E_{s n}=s \sqrt{n} \hbar \omega_{c}$. Moreover, the Fermi functions appearing in Eq. (7) are the equilibrium ones, and repeating the procedure of Sec. II leads directly to the result given by Eq. (15).

As for the evaluation of $\sigma_{x x}^{\text {col }}$, now there are no restrictions on the intralevel scattering, i.e., $n^{\prime}=n$ is allowed, while $n^{\prime} \neq n$ is forbidden for elastic scattering, since the eigenvalues do not depend on $k_{x}$. The form factors are

$$
\left|F_{n n}^{s s}(u)\right|^{2}=e^{-u}\left[L_{n}^{-1}(u)\right]^{2} / 4 .
$$

The form factors for the $n=0$ level must be considered separately; the result is $\left|F_{00}^{s s}\right|^{2}=e^{-u}$. For $\delta$-function impurities, or $k_{s} \gg q$ in $U(\mathbf{q})=U_{0} /\left(q^{2}+k_{s}^{2}\right)^{1 / 2}$, the conductivity $\sigma_{x x}^{\text {col can }}$ be evaluated analytically. We have

$$
\sigma_{x x}^{\mathrm{col}}=\frac{e^{2}}{h} \frac{\beta N_{I} U_{0}^{2}}{8 u_{c s} \hbar \omega_{c}} \sum_{s, n} 6 n f\left(E_{s n}\right)\left[1-f\left(E_{s n}\right)\right],
$$

where we use the relation

$$
\int_{0}^{\infty} u e^{-u}\left[L_{n}^{-1}(u)\right]^{2} d u=6 n
$$

that can be derived using the orthogonality of the Laguerre polynomials and their recurrence relation, ${ }^{38}(n+1) L_{n+1}(u)-$ $(2 n+1-u) L_{n}(u)+n L_{n-1}(u)=0$.

The main difference with the usual IQHE in semiconductors is the presence of the factor $6 n$ instead of $2 n+1$ in Eq. (28) (see Ref. 39). At low temperatures, the product $\beta f\left(E_{s n}\right)[1-$ $\left.f\left(E_{s n}\right)\right]$ in Eq. (28) can be replaced by a $\delta$ function or a Lorentzian; we adopt the former case and evaluate the longitudinal resistivity $\rho_{x x}$ from

$$
\rho_{x x}=\sigma_{x x} /\left[\sigma_{x x}^{2}+\sigma_{y x}^{2}\right] .
$$

In Fig. 2 we show the longitudinal resistivity $\rho_{x x}$ as a function of the electron concentration $n_{e}$ for finite Hall field $E_{H}=1 \mathrm{kV} \mathrm{cm}^{-1}$ (black solid curve) and for $E_{H}=0$ (red dashed curve). The other parameters are $A=40$, screening wave vector $k_{s}=10^{7} \mathrm{~m}^{-1}$, and impurity concentration $n_{i}=$ $5 \times 10^{10} \mathrm{~cm}^{-2}$. The magnetic field strength is $B=14 \mathrm{~T}$. The resistivity oscillates with the electron concentration as expected. A finite Hall field affects mainly the position of the peaks, while the values of the peaks are comparable for $n>1$.

In the left panel of Fig. 3, we show $\sigma_{x x}$ as a function of the electron concentration $n_{e}$ for $E=1 \mathrm{kV} \mathrm{cm}^{-1}$. The Fermi level is determined from $n_{e}$. The impurity concentration $n_{i}=5 \times 10^{10} \mathrm{~cm}^{-2}$, the screening wave vector $k_{s}=10^{7} \mathrm{~m}^{-1}$, and the parameter $A=40$ were chosen to obtain values of $\rho_{x x}$ compatible with the experiments. ${ }^{1}$ The absence of the peak, when the Fermi level is at the Dirac point $\left(n_{e} \approx 0\right)$, may probably be explained by a breakdown (of the $\mathrm{QHE}$ ) mechanism. 

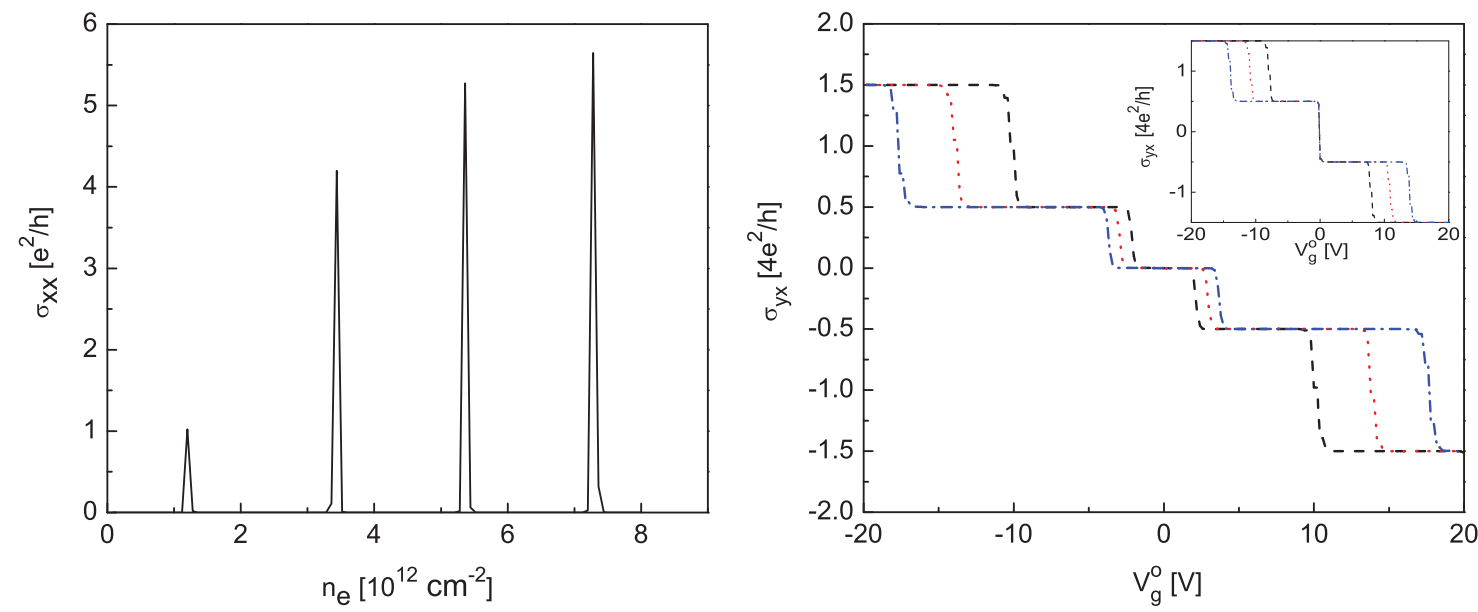

FIG. 3. (Color online) Left panel: Conductivity $\sigma_{x x}$ vs concentration. Right panel: Hall conductivity $\sigma_{x y}$ vs the gate voltage $V_{g}^{o}$ for three values of $B: 8 \mathrm{~T}$ (black, dashed curve), $11 \mathrm{~T}$ (red, dotted curve), and $14 \mathrm{~T}$ (blue, dash-dotted curve). The values of $\sigma_{x y}$ without the correction $(C=0)$ due to impurities are shown in the inset.

\section{LIFTING OF THE LEVEL DEGENERACY}

Recently there has been experimental evidence ${ }^{40,41}$ of the lifting of the fourfold degeneracy of the zeroth $(n=0)$ Landau level (LL) and partial lifting of the first $(n=1)$ LL for $B>20 \mathrm{~T}$ in samples having high mobility. ${ }^{12}$ Detailed magnetotransport studies ${ }^{41}$ of the activation gap may provide a hint regarding the origin of a particular splitting: For instance, $\Delta E(v= \pm 1)$ behaves like $\sqrt{B}$, suggesting a nonspin origin (possibly an electron-electron interaction and/or scattering by impurities $)$, while $\Delta E(v= \pm 4)$ has a linear dependence on the magnetic field (possibly a Zeeman-like splitting). The relation of the activation gap $\Delta E(v)$ to the actual splitting $\Delta \bar{E}(v)$ is not straightforward, since it also depends on the level width $\Gamma$; it is given by Ref. 42 as $\Delta E(v)=\Delta \bar{E}(v)-2 \Gamma$. Within this picture, $\Delta E(v= \pm 1)$ is expected ${ }^{40}$ to be on the scale of $e^{2} / \epsilon l_{c}$, hence proportional to $\sqrt{B}$. On the other hand, it would be natural to assume that the splitting $\Delta \bar{E}(v= \pm 1)$ is proportional to $\Gamma$ within the model proposed in this paper. Note that $\Gamma$ is also proportional ${ }^{2}$ to $\sqrt{B}$. Since the broadening $\Gamma$ is directly proportional to the impurity potential, it can be proven (see Appendix A) that the correction to the one-electron eigenvalue, $\Delta \bar{E}_{s n}=\langle s n|U(r)| s n\rangle$, is given by

$$
\begin{aligned}
\Delta \bar{E}_{s n}= & \frac{s C e^{-\xi^{2}}}{2^{n+1} n ! \sqrt{\pi}}\left[H_{n}^{2}(\xi)+2 n H_{n-1}^{2}(\xi)\right. \\
& \left.+2 \sqrt{2 n} \eta s H_{n}(\xi) H_{n-1}(\xi)\right],
\end{aligned}
$$

where $\xi=k_{x} l_{c}$ and $C=V_{0} / l_{c} L_{y}$ for short-range impurities; for long-range impurities, the value of $C$ is a bit more complicated (see Appendix A). As usual, the zeroth level should be treated separately; we find

$$
\Delta \bar{E}_{s 0}=s C e^{-\xi^{2}} / \sqrt{\pi} .
$$

The wave vector $k_{x}$ is of the order of $10^{6} \mathrm{~m}^{-1}$. We show the longitudinal conductivity $\sigma_{x x}$ in the left panel of Fig. 3 and the Hall conductivity $\sigma_{x y}$ in the right panel. We show $\sigma_{x x}$ as a function of the electron concentration. As seen, the first peak is shifted away to $n_{e}^{\prime}=1.15 \times 10^{12} \mathrm{~cm}^{-2}$ due to the correction $\Delta \bar{E}_{s n}$; without this correction, this peak would occur at $n_{e 0}=1 \times 10^{12} \mathrm{~cm}^{-2}$.

The Hall conductivity $\sigma_{x y}$ is shown for three different values of the magnetic field $B: 8 \mathrm{~T}$ (black, dashed curve), $11 \mathrm{~T}$ (red, dotted curve), and $14 \mathrm{~T}$ (blue, dash-dotted curve). The lifting of the LL degeneracy, due to impurity scattering, has been taken into account. The case when the LLs are still degenerate $(C=0)$ is shown in the inset. All other parameters are the same as in Fig. 2 for nonzero Hall field $\left(E_{H}=1 \mathrm{kV} \mathrm{cm}^{-1}\right)$. The influence of the energy correction $\left(\Delta \bar{E}_{s n}\right)$ is mostly pronounced at the Dirac point $\left(V_{g}^{0} \approx 0 \mathrm{~V}\right)$, where the appearance of the $v=0$ plateau is visible. This is so because the $n=0 \mathrm{LL}$ occurs at zero energy and its position depends on the density $n_{e}$, as shown in Fig. 4, where we plot the Fermi level versus $B$ for different densities $n_{e}$. At this point, we may compare the result for the $n=0$ plateau with that of Ref. 17 , which reads $\delta v_{\text {even }} \sim\left(n_{\text {imp }}\right)^{0.4}$, while ours varies as $\left(n_{\mathrm{imp}}\right)^{0.5}$. In fact, the values of $\Delta \bar{E}_{s n}$

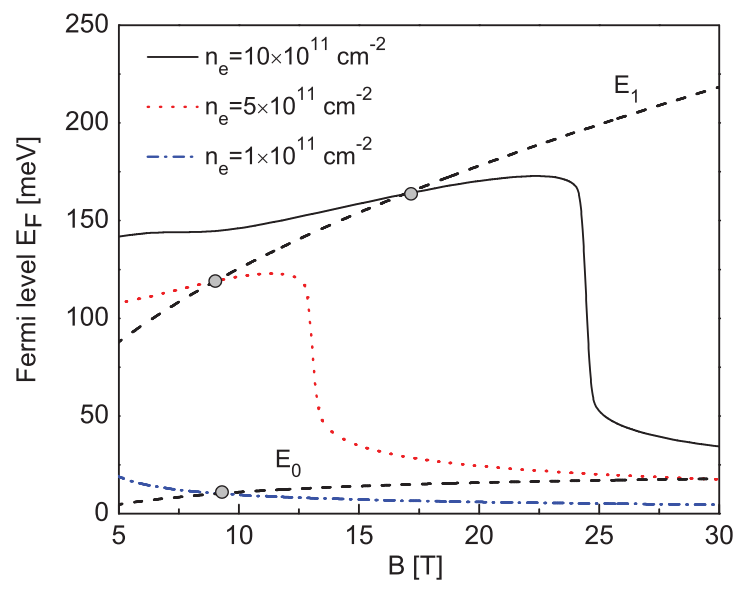

FIG. 4. (Color online) Fermi level vs magnetic field for three different concentrations: $n_{e}=10^{11} \mathrm{~cm}^{-2}$ (blue, dash-dotted curve), $n_{e}=5 \times 10^{11} \mathrm{~cm}^{-2}$ (red, dotted curve), and $n_{e}=10^{12} \mathrm{~cm}^{-2}$ (black, solid curve). The dashed curves show the Landau levels $n=0,1$ and the gray dots show their intersections with the Fermi level. 

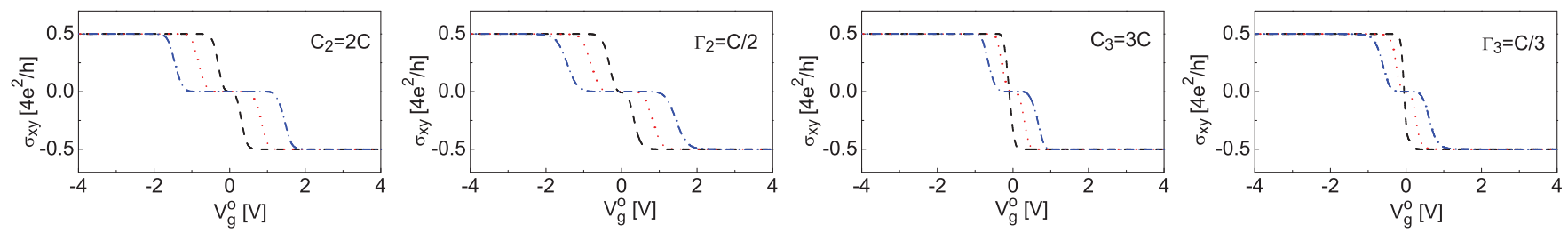

FIG. 5. (Color online) The region of the $n=0$ plateau for various values of the parameters $C$ and $\Gamma$. The two right panels are for $C=\Gamma$, given by Eq. (17), with $A=40, \Gamma_{2}=C / 2$, and $\Gamma_{3}=C / 3$; the two left panels are for fixed broadening $\Gamma, C_{2}=2 C$, and $C_{3}=3 C$. The dashed, dotted, and dash-dotted curves are for $B=8,11$, and 14 T, respectively.

for the $n=0,1,2,3$ levels, at $B=14 \mathrm{~T}$, are 11.7, 11.4, 5.5, and $4 \mathrm{meV}$, respectively. Correspondingly, assuming that any scattering other than that by impurities is negligible, we expect the plateaus of these levels to remain essentially unaffected for temperatures, such that $k_{B} T \ll \Delta \bar{E}_{s n}+\Gamma$, which yields $T \ll 200 \mathrm{~K}$. Notice that the limit for $T$ depends on the strength of the magnetic field, the impurity density, etc.

We plot $\sigma_{x y}$ versus the gate voltage $V_{g}^{0}$ in order to have a clearer contrast with the experimental data in Ref. 43. Note that $V_{g}^{0}$ is proportional to the concentration $n_{e}$ through the relation ${ }^{6} n_{e}=\left(\epsilon \epsilon_{0} / t e\right) \times V_{g}^{0}$, where $\epsilon$ and $\epsilon_{0}$ are the permittivities of the vacuum and $\mathrm{SiO}_{2}$, respectively, $e$ is the elementary charge, and $t=300 \mathrm{~nm}$ is the thickness of the $\mathrm{SiO}_{2}$ substrate, as commonly assumed. ${ }^{6,44}$ The results of Fig. 3 are in accordance with the experimental data; for instance, our calculation for $B=8 \mathrm{~T}$ gives the end of the $n=0$ plateau at $V_{g}^{0} \approx 10 \mathrm{~V}$, as in Ref. 43 , while for $B=14 \mathrm{~T}$ and the same plateau, our calculation gives $n_{e}=0.9 \times 10^{12} \mathrm{~cm}^{-2}$, while experimentally ${ }^{1}$ it is at $n_{e}^{\prime} \approx 1.1 \times 10^{12} \mathrm{~cm}^{-2}$.

The widths of the plateaus depend on the values of $C$, which appear in $\Delta \bar{E}_{s n}$, and the LL-width $\Gamma$, both of which are sample dependent. It is evident that with $C=0$, there are no plateaus around $V_{g}^{0}=0$. For $C \neq 0$, we have plateaus whose widths depend weakly on $\Gamma$, with $\Gamma=C$ giving the widest plateau. We show this with the two sets of panels in Fig. 5, and by focusing only on the $n=0$ plateau. The left set is for fixed $\Gamma[A=40$, see Eq. (17)] and $C$ progressively larger, while the right set is for fixed $C$ and $\Gamma$ progressively smaller. The different curves are marked as those in Fig. 3.

\section{CONCLUDING REMARKS}

We investigated the influence of the Hall field $E_{H} \neq 0$ and of the electron scattering by impurities on the Hall $\left(\sigma_{y x}\right)$ and longitudinal $\left(\sigma_{x x}\right)$ conductivities in graphene, mostly at low temperatures. We also considered the usual case, $E_{H}=0$.

For the evaluation of $\sigma_{y x}$, we used the Kubo-Greenwood formula for $\Gamma=0$, and obtained the well-known expression $\sigma_{y x}=4(N+1 / 2) e^{2} / h$ [cf. Eq. (15)]. With a slight modification of this formula, we also evaluated corrections to $\sigma_{y x}$ due to broadening $(\Gamma \neq 0)$ and the relative deviation from the $\Gamma=0$ case [see Eq. (16)].

An essential ingredient of the calculation of $\sigma_{y x}$ was the inclusion of the correction $\Delta \bar{E}_{s n}$ of the energy levels given by Eq. (31). This value depends on the strength of the scattering and lifts the $k_{x}$ degeneracy of the LLs as shown by Eq. (31). It is the main degeneracy lifting for zero Hall field $E_{H}=0$, but it also contributes to $E_{H} \neq 0$ when $\Delta \bar{E}_{s n}$ is added to
Eq. (4). This inclusion and the symmetry between electrons and holes gives rise to the $n=0$ Hall plateau. This correction or dispersion is embodied in the parameter $C$ and is absent for $C=0$, as the inset to the right panel of Fig. 3 makes clear. For $C \neq 0$, the plateau depends weakly on the value of $\Gamma$ (cf. Fig. 5). Usually $\Delta \bar{E}_{s n}$ is neglected, but in this particular case, where the $n=0, \mathrm{LL}$ is at zero energy; its contribution is paramount for the existence of the $n=0$ plateau but not for the $n>0$ plateaus, which are simply shifted a bit (cf. Fig. 3). Since $\Delta \bar{E}_{s n}$ decreases with $n$, this further makes the relative shift of the $n>0$ plateaus less important. Notice, though, that these $n>0$ plateaus are not split by the inclusion of $\Delta \bar{E}_{s n}$.

Accounting for the $n=0$ plateau due to scattering by impurities is in line with numerical calculations ${ }^{45}$ that consider electron-electron interaction as a mechanism for lifting the $k_{x}$ degeneracy of the LLs. Notice, though, that these numerical calculations are limited to the $n=0$ level, whereas ours are not. Although we used, we believe, realistic values for the parameters $C$ and $\Gamma$, a relative uncertainty in their true values still remains. One can expect that considering electronelectron interaction in a mean-field fashion, e.g., in the Hartree approximation, simply "renormalizes" the values of $C$ and $\Gamma$.

Since we considered only scattering by impurities, the results are valid, in general, only for low-temperatures $T$. However, since $\hbar \omega_{c}$ is much larger than in conventional semiconductors, we expect this temperature range to be significantly wider. A more specific limitation results from the inequality $k_{B} T \ll \Delta \bar{E}_{s n}+\Gamma$, which makes the upper limit in $T$ depend on the impurity density and the magnetic field. As to whether short-range or long-range scattering by impurities is the most important, the answer is unclear since the relevant parameters are not well known (see Sec. IV) and, at least in theory, the transport properties are insensitive to the potential range within the self-consistent Born approximation. ${ }^{2,20}$ It appears, though, that most observations and independent treatments favor long-range Coulomb impurities. ${ }^{17,18}$

For the evaluation of $\sigma_{x x}$, we used a standard linearresponse formula ${ }^{27}$ and also contrasted the results [e.g., Eq. (28)] with the well-known ones pertaining to the usual $\mathrm{IQHE}^{39,42}$ for elastic scattering by impurities. In either case, $\sigma_{x x}$ oscillates with electron concentration. The peak values depend on the details of the scattering potential when the Hall field $E_{H}$ is absent; if it is present and sufficiently weak so that it affects only the scattering and not the Fermi functions, we have an additional dependence (see Fig. 2). This behavior of $\sigma_{x x}$ is also reflected in that of the longitudinal resistivity $\rho_{x x}$. In all results, we neglected the influence of the Zeeman term. We have verified, though, that at least for the $B$ fields we considered, $B \leqslant 20 \mathrm{~T}$, this term can be safely neglected. 


\section{ACKNOWLEDGMENTS}

We thank Dr. O. Balev for useful exchanges regarding the results of Sec. II. Our work was supported by the Canadian NSERC Grant No. OGP0121756.

\section{APPENDIX A: DISPERSION OF THE ENERGY LEVELS}

Below we evaluate the $k_{x}$ corrections to the eigenvalues due to scattering by randomly distributed impurities.

(1) Short-range impurities. The potential has the form $U(\mathbf{r})=V_{0} \delta(\mathbf{r})=V_{0} \delta(x) \delta(y)$, with $V_{0}$ the strength of the potential. Then, the correction to the eigenvalues, $\Delta E_{s n}=$ $\left\langle s n k_{x}|U(r)| s n k_{x}\right\rangle$, is given by Eq. (31). If we replace $L_{y}$ in Eq. (31) by the average distance between the impurities $\left(\propto 1 / \sqrt{n_{i}}\right)$, as done in Ref. 2, we obtain

$$
C \approx \Gamma=\sqrt{n_{i} / 2 \pi} U_{0} / k_{s} l_{c}
$$

Then, Eq. (17) gives, for $n_{i} \approx 10^{10} \mathrm{~cm}^{-2}$ and $k_{s} \approx 2 \times$ $10^{8} \mathrm{~cm}^{-1}$, the value $A \approx 90$, which is in accordance with Ref. 29 where $A$ lies in the range 50-100. For smaller $k_{s}$, giving $A=40$ and $B=14 \mathrm{~T}$, one finds $\Delta E_{0}=11.7 \mathrm{meV}$.

(2) Screened Coulomb impurities. The potential has the form $U(\mathbf{r})=U_{0} e^{-k_{s} r} / r$, with $U_{0}=e^{2} / 4 \pi \epsilon_{0} \epsilon_{r}$ and Fourier transform $U(q)=U_{0} l_{c} / \sqrt{2\left(u+u_{c s}\right)}$. For $E_{H}=0$, we find

$$
\begin{aligned}
\Delta E_{s n}= & \frac{U_{0}}{2 \sqrt{2} l_{c}} \int_{0}^{\infty} d u e^{-u / 2} \cos (\xi \sqrt{2 u}) \\
& \times\left[L_{n}(u)+L_{n-1}(u)\right] / \sqrt{u+u_{c s}} .
\end{aligned}
$$

For $B=8 \mathrm{~T}$, Eq. (A2) gives the numerical values $\Delta E_{i} \approx$ $8,24,12 \mathrm{meV}(i=0,1,2)$ for the correction to the first three levels. Using the expansions $\cos (\xi \sqrt{2 u}) \approx 1-\xi^{2} u+\xi^{4} u^{2} / 6$ and $\left(u+u_{c s}\right)^{-1 / 2} \approx u_{c s}^{-1 / 2}\left(1-u / 2 u_{c s}\right)$, one finds

$$
\begin{aligned}
\Delta E_{s n} \approx & \frac{U_{0}}{l_{c} \sqrt{2 u_{c s}}}\left[I_{n}^{(0)}-\left(1 / 2 u_{c s}+\xi^{2}\right) I_{n}^{(1)}\right. \\
& \left.+\left(\xi^{4} / 6+\xi^{2} / 2 u_{c s}\right) I_{n}^{(2)}-\left(\xi^{4} / 12 u_{c s}\right) I_{n}^{(3)}\right],
\end{aligned}
$$

with $I_{n}^{(m)}=(2 m-1) ! ! \sqrt{2 \pi} F(-n, m+1 / 2)$ and $F(\ldots)$ the hypergeometric functions. ${ }^{38}$ For $n=0$, we have $I_{0}^{(0)}=$ $\sqrt{2 \pi}, I_{0}^{(1)}=\sqrt{2 \pi}, I_{0}^{(2)}=3 \sqrt{2 \pi}$, and $I_{0}^{(3)}=15 \sqrt{2 \pi}$.

\section{APPENDIX B: CORRECTIONS TO $\sigma_{y x}^{n d}$ DUE TO BROADENING}

We multiply and divide the summand of Eq. (7) by $E_{\zeta}-$ $E_{\zeta^{\prime}}-i \Gamma_{\zeta}$. If $\Gamma_{\zeta}=\Gamma$ is the same for all $\zeta$, the resulting term proportional to $\Gamma$ vanishes and Eq. (7) takes the form

$$
\sigma_{y x}^{n d}=\frac{i \hbar e^{2}}{S_{0}} \sum_{\zeta, \zeta^{\prime}} \frac{\left(f_{\zeta}-f_{\zeta^{\prime}}\right) v_{v \zeta \zeta^{\prime}} v_{\mu \zeta^{\prime} \zeta}}{\left(E_{\zeta}-E_{\zeta^{\prime}}\right)^{2}+\Gamma^{2}} .
$$

The difference $E_{\zeta}-E_{\zeta^{\prime}}$ is of the order of $\hbar \omega_{c}$ [cf. Eq. (4)]. For small $\Gamma \ll \hbar \omega_{c}$, the denominator can be expanded in powers of $\left(\Gamma / \hbar \omega_{c}\right)^{2}=2 / A$ [see Eq. (17)]; this leads to

$$
\sigma_{y x}^{n d} \cong \frac{i \hbar e^{2}}{S_{0}} \sum_{\zeta, \zeta^{\prime}} \frac{\left(f_{\zeta}-f_{\zeta^{\prime}}\right) P_{\zeta \zeta^{\prime}}}{\left(E_{\zeta}-E_{\zeta^{\prime}}\right)^{2}}(1-2 / A),
$$

where $P_{\zeta \zeta^{\prime}}$ is the product of the velocities given by Eq. (10). The sum over momenta gives a factor $S_{0} e B / h$. Taking into account the degeneracy $g_{s}=4$, the correction $\Delta \sigma_{y x}^{n d}$ to the Hall conductivity $\sigma_{y x}^{n d}$ takes the form

$\Delta \sigma_{y x}^{n d} \cong-\frac{e^{2} g_{s}}{4 A \gamma^{5 / 2}} \sum_{n n^{\prime} s s^{\prime}} \frac{\left(f_{n s}-f_{n^{\prime} s^{\prime}}\right)\left(\delta_{n-1, n^{\prime}}-\delta_{n, n^{\prime}-1}\right)}{\left(\sqrt{n} s-\sqrt{n^{\prime}} s^{\prime}\right)^{4}}$.

If we treat separately the sums between the "+" and "+" branches, "+" and "-" branches, "-" and "+" branches, etc., some terms will cancel out and lead to

$$
\begin{aligned}
\Delta \sigma_{y x}^{n d} \cong & -\frac{32 e^{2}}{A \gamma^{5 / 2} h} \sum_{n}\left(n^{2}+n+1 / 8\right) \\
& \times\left(f_{n}^{+}-f_{n+1}^{+}+f_{n}^{-}-f_{n+1}^{-}\right) .
\end{aligned}
$$

For small temperatures, this expression turns into

$$
\Delta \sigma_{y x}^{n d} \cong-\frac{32 e^{2}}{A \gamma^{5 / 2} h}\left(N^{2}+N+1 / 8\right),
$$

where $N$ is the largest integer contained in $E_{F} / \hbar \omega_{c}$. The relative deviation from the $\Gamma=0$ case is then

$$
\frac{\Delta \sigma_{y x}^{n d}}{\sigma_{y x}^{n d}} \cong-\frac{8}{A \gamma^{3 / 2}} \frac{N^{2}+N+1 / 8}{N+1 / 2} .
$$

For $N=0$ and $\gamma \approx 1$, it has the very simple form

$$
\frac{\Delta \sigma_{y x}^{n d}}{\sigma_{y x}^{n d}} \cong-\frac{2}{A} \text {. }
$$

If the impurity scattering is weak, the dimensionless parameter $A$ (Ref. 2 ) has a high value, $A \approx 100$. Then for $B=14 \mathrm{~T}$, we have $\Gamma \approx 20 \mathrm{meV}$, while $\hbar \omega_{c}=149 \mathrm{meV}$, and the deviation of the Hall conductivity is about $2 \%$. If we take $A \approx 40$, the deviation is about $4 \%$.

\footnotetext{
${ }^{1}$ A. H. Castro Neto, F. Guinea, N. M. R. Peres, K. S. Novoselov, and A. K. Geim, Rev. Mod. Phys. 81, 109 (2009).

${ }^{2}$ Y. Zheng and T. Ando, Phys. Rev. B 65, 245420 (2002).

${ }^{3}$ V. P. Gusynin and S. G. Sharapov, Phys. Rev. Lett. 95, 146801 (2005).

${ }^{4}$ N. M. R. Peres, F. Guinea, and A. H. Castro Neto, Phys. Rev. B 73, 125411 (2006).
}

${ }^{5}$ A. M. J. Schakel, Phys. Rev. D 43, 1428 (1991).

${ }^{6} \mathrm{~K}$. S. Novoselov, A. K. Geim, S. V. Morozov, D. Jiang, M. I. Katsnelson, I. V. Grigorieva, S. V. Dubonos, and A. A. Firsov, Nature (London) 438, 197 (2005).

${ }^{7}$ Y. Zhang, Y.-W. Tan, H. L. Stormer, and P. Kim, Nature (London) 438, 201 (2005). 
${ }^{8}$ K. S. Novoselov, Z. Jiang, Y. Zhang, S. V. Morozov, H. L. Stormer, U. Zeitler, J. C. Maan, G. S. Boebinger, P. Kim, and A. K. Geim, Science 315, 1379 (2005).

${ }^{9}$ S. Masubuchi, K. Suga, M. Ono, K. Kindo, S. Takeyama, and T. Machida, J. Phys. Soc. Jpn. 77, 113707 (2008).

${ }^{10} \mathrm{X}$. Wu, Y. Hu, M. Ruan, N. K. Madiomanana, J. Hankinson, M. Sprinkle, C. Berger, and W. A. de Heer, Appl. Phys. Lett. 95, 223108 (2009).

${ }^{11}$ D. L. Miller, K. D. Kubista, G. M. Rutter, M. Ruan, W. A. de Heer, P. N. First, and J. A. Stroscio, Science 324, 924 (2009).

${ }^{12}$ Z. Jiang, Y. Zhang, Y.-W. Tan, H. L. Stormer, and P. Kim, Solid State Commun. 143, 14 (2007).

${ }^{13}$ Y. Zhang, Z. Jiang, J. P. Small, M. S. Purewal, Y.-W. Tan, M. Fazlollahi, J. D. Chudow, J. A. Jaszczak, H. L. Stormer, and P. Kim, Phys. Rev. Lett. 96, 136806 (2006).

${ }^{14}$ V. P. Gusynin and S. G. Sharapov, Phys. Rev. Lett. 95, 146801 (2005); Phys. Rev. B 73, 245411 (2006).

${ }^{15}$ A. H. Castro Neto, F. Guinea, and N. M. R. Peres, Phys. Rev. B 73, 205408 (2006).

${ }^{16}$ I. F. Herbut, Phys. Rev. B 75, 165411 (2007).

${ }^{17}$ P. M. Ostrovsky, I. V. Gornyi, and A. D. Mirlin, Phys. Rev. 77, 195430 (2008).

${ }^{18}$ T. Champel and S. Florens, Phys. Rev. B 82, 045421 (2010).

${ }^{19}$ T. Kramer, C. Kreisbeck, V. Krueckl, E. J. Heller, R. E. Parrott, and C.-T. Liang, Phys. Rev. B 81, 081410(R) (2010).

${ }^{20}$ M. Koshino and T. Ando, Phys. Rev. B 75, 033412 (2007).

${ }^{21}$ M. E. Raikh and T. V. Shahbazyan, Phys. Rev. B 47, 1522 (1993).

${ }^{22}$ O. G. Balev and P. Vasilopoulos, Phys. Rev. B 47, 16410 (1993).

${ }^{23}$ P. S. Zyryanov and M. Klinger, Quantum Theory of Electron Transport Phenomena in Crystalline Semiconductors [in Russian] (Nauka, Moscow, 1976).

${ }^{24}$ A. H. MacDonald, T. M. Rice, and W. F. Brinkman, Phys. Rev. B 28, 3648 (1983).

${ }^{25}$ V. Lukose, R. Shankar, and G. Baskaran, Phys. Rev. Lett. 98, 116802 (2007).

${ }^{26}$ N. M. R. Peres and E. V. Castro, J. Phys. C 19, 406231 (2007).
${ }^{27}$ M. Charbonneau, K. M. Van Vliet, and P. Vasilopoulos, J. Math. Phys. 23, 318 (1982); P. Vasilopoulos, Phys. Rev. B 32, 771 (1985).

${ }^{28}$ O. G. Balev and P. Vasilopoulos, Phys. Rev. B 54, 4863 (1996).

${ }^{29}$ T. Ando, J. Phys. Soc. Jpn. 75, 074716 (2006).

${ }^{30}$ N. M. R. Peres, J. M. B. Lopes dos Santos, and T. Stauber, Phys. Rev. B 76, 073412 (2007).

${ }^{31}$ E. Fradkin, Phys. Rev. B 33, 3257 (1986).

${ }^{32}$ A. W. W. Ludwig, M. P. A. Fisher, R. Shankar, and G. Grinstein, Phys. Rev. B 50, 7526 (1994).

${ }^{33}$ S. Adam, E. H. Hwang, V. M. Galitski, and S. D. Sarma, Proc. Natl. Acad. Sci. USA 104, 18392 (2007).

${ }^{34}$ K. Nomura and A. H. MacDonald, Phys. Rev. Lett. 98, 076602 (2007).

${ }^{35}$ J.-H. Chen, G. Jang, S. Adam, M. S. Fuhrer, E. D. Williams, and M. Ishigami, Nature Phys. 4, 377 (2008).

${ }^{36}$ Strictly speaking, for $E \neq 0$, there is a Drude type contribution to the conductivity $\sigma^{\mathrm{dif}}$, stemming from the second term in Eq. (4) or the term $\propto \delta_{n, n^{\prime}}$ in Eq. (8) (see Ref. 27). However, this $\sigma^{\text {dif }}$ is about five orders of magnitude smaller than $\sigma^{\text {col }}$ evaluated in the text and is therefore neglected. For $E=0$, though, this $\sigma^{\mathrm{dif}}$ vanishes.

${ }^{37}$ C. H. Lewenkopf, E. R. Mucciolo, and A. H. Castro Neto, Phys. Rev. B 77, 081410(R) (2008).

${ }^{38}$ I. S. Gradshteyn and I. M. Ryzhik, Table of Integrals, Series, and Products (Academic, Orlando, 1980).

${ }^{39}$ P. Vasilopoulos and C. M. Van Vliet, Phys. Rev. B 34, 1057 (1986).

${ }^{40}$ Z. Jiang, Y. Zhang, H. L. Stormer, and P. Kim, Phys. Rev. Lett. 99, 106802 (2007).

${ }^{41}$ A. J. M. Giesbers, L. A. Ponomarenko, K. S. Novoselov, A. K. Geim, M. I. Katsnelson, J. C. Maan, and U. Zeitler, Phys. Rev. B 80, 201403(R) (2009).

${ }^{42}$ T. Ando, A. B. Fowler, and F. Stern, Rev. Mod. Phys. 54, 437 (1982).

${ }^{43}$ J. G. Checkelsky, L. Li, and N. P. Ong, Phys. Rev. Lett. 100, 206801 (2008).

${ }^{44}$ S. Cho and M. S. Fuhrer, Phys. Rev. B 77, 081402(R) (2008).

${ }^{45}$ Y. Barlas, R. Côté, K. Nomura, and A. H. MacDonald, Phys. Rev. Lett. 101, 097601 (2008). 